\title{
Micro-NRA and micro-3HIXE with He-3 microbeam on samples exposed in ASDEX Upgrade and Pilot-PSI machines
}

\section{Kelemen, Mitja}

2017-08-01

Kelemen, M , Zaloznik , A, Vavpetic , P , Pecovnik, M , Pelicon, P , Hakola , A, Lahtinen , A , Karhunen , J , Piip , K , Paris , P , Laan , M , Krieger , K, Oberkofler , M , van der Meiden , H, Markelj , S \& ASDEX Upgrade Team 2017 , ' Micro-NRA and micro-3HIXE with He-3 microbeam on samples exposed in ASDEX Upgrade and Pilot-PSI machines ' , Nuclear Instruments \& Methods in Physics Research. Section B: Beam Interactions with Materials and Atoms , vol. 404 , pp. 179-184 . https://doi.org/10.1016/j.nimb.2017.01.072

http://hdl.handle.net/10138/308515

https://doi.org/10.1016/j.nimb.2017.01.072

cc_by_nc_nd

acceptedVersion

Downloaded from Helda, University of Helsinki institutional repository.

This is an electronic reprint of the original article.

This reprint may differ from the original in pagination and typographic detail.

Please cite the original version. 


\section{Micro-NRA and micro-3HIXE with ${ }^{3} \mathrm{He}$ microbeam on samples exposed in ASDEX Upgrade and pilot-PSI machines}

Mitja Kelemen ${ }^{1,2}$, Anže Založnik ${ }^{1}$, Primož Vavpetič ${ }^{1}$, Matic Pečovnik ${ }^{1}$, Primož Pelicon ${ }^{1}$, Antti Hakola ${ }^{3}$, Aki Lahtinen ${ }^{4}$, Juuso Karhunen ${ }^{5}$ Kaarel Piip ${ }^{6}$, Peeter Paris $^{6}$, Matti Laan ${ }^{6}$, Karl Krieger $^{7}$, Martin Oberkofler ${ }^{7}$, the ASDEX Upgrade Team ${ }^{7}$, Hennie van der Meiden ${ }^{8}$ and Sabina Markelj ${ }^{1}$

${ }^{1}$ Jožef Stefan Institute, Jamova 39, SI-1000 Ljubljana, Slovenia

2 Jozef Stefan International Postgraduate School, Jamova cesta 39, 1000 Ljubljana, Slovenia

${ }^{3}$ VTT Technical Research Centre of Finland Ltd, P O Box 1000, FI-02044, VTT, Finland

${ }^{4}$ University of Helsinki, Department of Physics, P O Box 64, FI-00014, University of Helsinki, Finland

${ }^{5}$ Aalto University, Department of Applied Physics, P O Box 11100, FI-00076, Aalto, Finland

${ }^{6}$ University of Tartu, Institute of Physics, W. Ostwaldi Str , 50411, Tartu, Estonia

${ }^{7}$ Max-Planck-Institut für Plasmaphysik, Boltzmannst. 2, D-85748 Garching, Germany

${ }^{8}$ Dutch Institute for Fundamental Energy Research, P.O. Box 6336, 5600 HH Eindhoven The Netherlands

\section{*Corresponding author: Mitja Kelemen}

E-mail address: mitja.kelemen@ijs.si 


\section{Abstract}

Micro nuclear reaction analysis (micro-NRA) exploiting the nuclear reaction $\mathrm{D}\left({ }^{3} \mathrm{He}, \mathrm{p}\right)^{4} \mathrm{He}$ was used for post-mortem analyses of special marker samples, exposed to deuterium plasma inside ASDEX Upgrade (AUG) tokamak and to the deuterium plasma jet in the Pilot-PSI linear plasma gun. Lateral concentration profiles of deuterium and erosion/deposition profiles of the marker materials were obtained by a combination of micro-NRA and particle induced $\mathrm{x}$-ray emission by ${ }^{3} \mathrm{He}$ beam (3HIXE). In the case of AUG samples, where $25 \mathrm{~nm}$ thick $\mathrm{W}$ marker layers had been deposited on unpolished and polished graphite substrates, the effect of surface roughness on local erosion and deposition was also investigated. The lateral distribution of $\mathrm{W}$ concentration showed that erosion is much more distinct in the case of polished samples and the resulting surface shows a "leopard" skin pattern of W accumulated on carbon aggregates left on the surface from polishing. The Pilot-PSI samples indicated preferential accumulation of deuterium a few $\mathrm{mm}$ off from the centre of the region affected by the plasma beam. This is connected with the largest surface modifications while the thick deposited layers at the centre do not favour deuterium retention per se. The results were cross correlated with those obtained using laser-induced breakdown spectroscopy (LIBS). With its quantitative abilities, micro-NRA provided essential calibration data for in situ LIBS operation, as well as for complementary post mortem Secondary Ion Mass Spectroscopy (SIMS).

Keywords: Focused ion beams, Deuterium, ${ }^{3} \mathrm{He}$, Nuclear Reaction Analysis, 3HIXE 


\section{Introduction}

One of the most critical issue in the construction of a thermonuclear reactor based on magnetic confinement in the tokamak configuration is the inner wall of the reactor, directly in contact with a hot plasma. During the operation of a fusion device, the wall is subjected to a combination of neutron and charged particle bombardment, large and uneven thermal loads, photon irradiation and neutral hydrogen exposure. This leads to erosion, deposition, adsorption of hydrogen and material lattice damage [1]. The processes at the surface of the material or in its bulk lead to the accumulation of fusion fuel (mainly hydrogen isotopes) in the vessel walls. To obtain a depth profile, up to $10 \mu \mathrm{m}$, of retained deuterium fuel in plasma-exposed samples, nuclear reaction analysis (NRA) is often used via the $D\left({ }^{3} \mathrm{He}, \mathrm{p}\right)^{4} \mathrm{He}$ reaction $[2,3]$.

Usually NRA is used in a broad beam configuration with beam diameter equal or even larger than $1 \mathrm{~mm}$ to obtain global and local erosion and deposition profiles of various elements on the wall structures in a rapid and straightforward manner $[4,5]$. In addition, wall structures can be characterized in situ, during their exposure to plasma [6], flux of atomic hydrogen [7, 8] or energetic $W$ ions simulating neutron damage [9].

At Jožef Stefan Institute (JSI) a different method has been developed. Here, a focused ${ }^{3} \mathrm{He}$ beam is used to investigate especially fuel retention on sample surfaces on the micrometer scale [10]. With such ${ }^{3} \mathrm{He}$ beam we can also measure spectra from Rutherford backscattering spectroscopy (RBS) which help us to study erosion and deposition phenomena on micro scale. Other chemical impurities, mostly heavier elements, can be located and measured via particle induced $\mathrm{x}$-ray emission 
(PIXE). The motivation to use micro-NRA for plasma-exposed samples relates to its unique ability to provide quantitative lateral distribution of deuterium $[11,12]$.

Tungsten or advanced tungsten alloys are materials of choice for high heat flux plasma-facing components in modern fusion reactors [13]. In the present study we report the micro-NRA results obtained for samples with thin $\mathrm{W}$ marker coatings and with different surface roughness that were exposed to D plasma discharges in the ASDEX Upgrade (AUG) tokamak [14]. This tokamak is equipped with divertor manipulator, that enables exposing several small samples to divertor plasmas in the vicinity of the low-field side strike point where the plasma-wall interaction processes are the strongest. The manipulator can be retracted after a single plasma shot without breaking the vacuum of the AUG vessel $[15,16]$.

In addition, we have applied micro-NRA on $\mathrm{W}+\mathrm{Y}$ coated samples that were exposed to D plasmas (with Ne boost) in the Pilot-PSI linear plasma gun $[17,18]$. The results have been cross-correlated with the data obtained using laser-induced breakdown spectroscopy (LIBS), which was executed at defined positions over the sample. LIBS is a method of high potential value for the control of the deuterium/tritium retention and elucidating the elemental composition of deposited layers inside of an operating tokamak device, as it can be executed in-situ during the operation of a fusion reactor. The elemental inventory was measured also with secondary ion mass spectrometry (SIMS) over a series of selected positions to determine the lateral concentration profiles over the plasma profile. The profiles obtained by a combination of micro-NRA and 3HIXE were compared to those of LIBS and SIMS [19]. With its quantitative abilities, micro-NRA provided essential calibration data for qualitative complementary methods LIBS and SIMS. 


\section{Experimental set-up}

Measurements with micro-beam were performed using the 2-MV tandem accelerator of the Jožef Stefan Institute. Using a micro-beam line, located at $-10^{\circ}$ from the exit of the accelerator. The micro-beam experimental line coupled with a high brightness multicusp ion source, is primarily used for micro-PIXE, where the proton beam is used and can be focused to dimensions of $500 \times 500 \mathrm{~nm}^{2}$ [20].

For NRA measurements we used a ${ }^{3} \mathrm{He}$ beam. Negative ${ }^{3} \mathrm{He}$ ion beam is formed in the combination of a duoplasmatron ion source and a Li- change exchange canal, involving a setup for ${ }^{3} \mathrm{He}$ and ${ }^{4} \mathrm{He}$ gas mixing within the duoplasmatron housing to spare precious ${ }^{3} \mathrm{He}$ gas. Positive $\mathrm{He}$ ions with the initial energy of $20 \mathrm{keV}$ are directed through a charge exchange channel, where collisions with Li vapour create short living $\mathrm{He}^{-}$ions. The ${ }^{3} \mathrm{He}^{2+}$ ions are accelerated with a tandem accelerator to an energy of 3.3 MeV. Under such conditions, a rectangular ${ }^{3} \mathrm{He}$ ion beam with a size of $10 \times 10 \mu \mathrm{m}^{2}$ is produced on a measurement position. Due to low brightness of the generated He beam both object and collimating slits at the microprobe have to be widely opened in order to achieve a particle current of $300 \mathrm{pA}$. The dimensions of beam are optimised with a knife edge method on a copper grid, using induced Ka Xray emission and i-Ge X-ray detector.

The end station (Figure 1) is equipped with a 5-axes manipulator and a microscope with a camera for samples positioning in the focal plane of the ion beam. In front of the end station a triplet of quadrupole magnetic lenses is used for focusing the ion beam and with deflection coils for manipulation of the beam in a raster pattern on the sample. With existing hardware, we are able to scan the beam across an area of $2.2 \times 2.2 \mathrm{~mm}^{2}$ and produce elemental maps with a resolution of $256 \times 256$ pixels. 
For dose normalization we use a beam chopper combined with an RBS detector [21]. The chopper is positioned after the collimating slits and before the scanning coils, cutting a fraction of the ion flux that is then directed onto the sample. In this way, we warrant the proportionality of the backscattering ions from the chopper blades detected by partially implanted passivated silicon (PIPS) detector and the primary ion dose deposited on the sample. A high-purity germanium X-ray detector is positioned at $135^{\circ}$ with respect to the beam direction. It is used to reveal the concentrations and distribution of various metallic impurities in the samples by particle induced $\mathrm{x}$-ray emission by ${ }^{3} \mathrm{He}$ beam (3HIXE) and PIXE measurements. A second X-ray detector, a $30-\mathrm{mm}^{2}$ Peltier-cooled silicon drift detector (SDD), is installed at $135^{\circ}$ with respect to the beam direction to detect low energy X-rays with energies from $700 \mathrm{eV}$ to $8 \mathrm{keV}$. To determine concentrations of marker elements and their depth profiles as well as to study possible layered structure of the analysed samples, a PIPS-type RBS detector with a 300 micrometer-thick depletion layer is positioned at $135^{\circ}$ with respect to beam direction, covering a solid angle of $5.6 \mathrm{msr}$. It is equipped with an 800-nm thick Al foil serving as a light block filter. For fuel retention and deposition investigations, an NRA detector was added to the standard detector configuration of the micro-beam end station (see Figure 1). For the detection of the fast protons $\mathrm{D}\left({ }^{3} \mathrm{He}, \mathrm{p}\right)^{4} \mathrm{He}$ nuclear reaction, we are using a PIPS detector with a 1000 micrometer-thick depletion layer and active area of $300 \mathrm{~mm}^{2}$. The NRA detector has a solid angle of $0.14 \mathrm{sr}$ and is positioned at $135^{\circ}$ with respect to the primary beam direction. To separate back-scattered ${ }^{3} \mathrm{He}$ from protons produced in nuclear reactions as well as to partly stop the protons and shield the detector against visible light, we inserted a $125 \mu \mathrm{m}$ thick kapton foil and a $6 \mu \mathrm{m}$ thick Al foil in front of it. The acquisition system is designed in the way that each detector 
event in the set of detectors is recorded and saved in a list mode together with information on the beam position.

\section{Measurements and results}

The samples were analysed by performing several scans (see section 2) along a selected line of interest.

First, two AUG samples with average $25 \mathrm{~nm}$ thick W layers. The samples are part of 2014 marker samples batch. The W layer is deposited on either unpolished or polished fine grain graphite substrate and its thickness and homogeneity of W was measured by ${ }^{4} \mathrm{He}$ RBS before mounting the samples on the AUG divertor manipulator, not by authors.

Samples were analysed after they had been exposed to 13 identical low-density Lmode deuterium plasma discharges in the vicinity of the low-field side strike point of AUG [16]. The analyses consisted of 7 rectangular analysing regions across a single line in the so-called poloidal direction at equidistant steps of $2.8 \mathrm{~mm}$; along this line the changes in the plasma conditions during the experiment were the largest. In this way we obtained good information on any eventual topographical effect on the surface. The scanned areas are shown on Figure 2a for the polished AUG sample and on Figure $2 \mathrm{~b}$ for the unpolished AUG sample.

The Pilot-PSI sample, for its part, was a $2 \mu \mathrm{m}$ thick $\mathrm{W}+\mathrm{Y}(\mathrm{Y}$ content $\sim 5$ at.\%) coating on Mo that had been exposed first to D+Ne plasma discharges for $300 \mathrm{~s}$ (to modify surface and obtain measurable erosion) and then to D plasmas for another $300 \mathrm{~s}$ to load the surface with as much plasma fuel as possible. For the $\mathrm{D}+\mathrm{Ne}$ mixture the volumetric flow rates at the plasma source were $0.7 \mathrm{slm}$ (standard liter per minute) $\mathrm{D}_{2}$ and $2.22 \mathrm{slm}$ Ne. Nevertheless, due to different diffusion coefficients and mechanisms the ratio may be different at the target. The volumetric flow rate for the 
pure D plasma was $2.5 \mathrm{slm}$. Typical plasma parameters were: the electron density of $1-4 \times 10^{20} \mathrm{~m}^{-3}$, the electron temperature 1-2 eV with particle flux of about $10^{24} \mathrm{~m}^{-2} \mathrm{~s}$. Usually, the plasma beam has Gaussian shape. Nevertheless, in case of the $\mathrm{Ne}+\mathrm{D}$ mixture the distribution was not Gaussian, but could be still characterized by FWHM. For the D+Ne the FWHM was $12 \mathrm{~mm}$ and for pure D plasma $25 \mathrm{~mm}$ (due to different confining magnetic field). The temperature distribution at the target followed Gaussian profile. The target surface temperature was up to $1300^{\circ} \mathrm{C}$ (around $600^{\circ} \mathrm{C}$ at the edge of the target) for the $\mathrm{D}+\mathrm{Ne}$ plasma and up to $600^{\circ} \mathrm{C}$ for the $\mathrm{D}$ plasma. Thus the particle fluence was in the range of $10^{26} \mathrm{~m}^{-2}$. For the pure $\mathrm{D}$ plasma flux was nearly 20 times weaker. The target was biased with $-40 \mathrm{~V}$, the ion energy at the target was around $38 \mathrm{eV}$.

In the micro beam measurements, the measured areas were shifted for $2 \mathrm{~mm}$. In this way, the consecutive frames partially overlapped, as shown in Figure 3, which allowed us to produce continuous elemental profile for that sample along a radial line across the exposed beam spot. To obtain a linear elemental profile of AUG and PSI sample along the line of interest, we projected data from 2D map on the line of interest. Obtained NRA and RBS spectra were analysed with the SIMNRA program [22] to calculate the concentrations and depth profiles of different elements.

The lateral distribution of $D$ and $W$ concentrations along the poloidal axis for AUG samples is shown in Figure 4. We can observe that the gross erosion of $\mathrm{W}$ is more pronounced on the polished graphite sample near the strike point. There we observe also increase of $D$ retention due to plasma implantation or due to co-deposition. This erosion is not so pronounced in the case of the unpolished graphite, due to the larger initial surface roughness. A more detailed view in the structure of this surface 
is given by micro-3HIXE in figure 5 . The yield for $W$ is now proportional to the concentration of $\mathrm{W}$ and, as all the samples have a thin coating of $\mathrm{W}$, we can neglect the effects of autoabsorption. We observe a large difference in the surface topography of polished and unpolished graphite sample. As can be seen in Figure 5 left, on the smooth-polished graphite sample tungsten is deposited on carbon aggregates forming a "leopard-skin" pattern. In the case of unpolished graphite sample, Figure 5 right, the $\mathrm{W}$ distribution more resembles to a snake skin. The existence of the aggregates and the snake-skin pattern is confirmed by scanning electron microscope (SEM) studies. The SEM images are presented in Figure 6 left for the polished and 6 right for the unpolished sample. The SEM measurements were done on approximately the same regions as micro-3HIXE measurements shown on Figure 5, as total correlation of areas measured with 3HIXE and SEM in current set ups is not possible. Overall the measurements are in line with expected values from broad-beam RBS and NRA measurements [16].

In the case of the Pilot-PSI sample we integrated the NRA and 3HIXE data collected in the list mode file, which is $2 \mathrm{D}$ distribution map, over perpendicular axis to radial direction of the plasma impact and therefore obtaining a linear elemental profile. Through this process we obtained a projection of elemental yields along the radial direction across the spot that the Pilot-PSI plasma jet had produced on the surface. The obtained profile of deuterium amount (see Figure 7) reveals deuterium depletion in the centre of the plasma impact, a slight increase in the concentration levels close to the edge of the impact crater induced by D-Ne discharge and uniform but low $D$ amounts in regions far away from the impact [19]. The $D$ is preferentially accumulated a few $\mathrm{mm}$ off from the centre of the region affected by the D-Ne plasma beam i.e. strike crater. This effect is connected with the largest surface modifications 
(the Ne beam that modifies the surface shows a hollow profile) and Gaussian shape of the temperature profile $\left(100{ }^{\circ} \mathrm{C}\right.$ for $\mathrm{D}-\mathrm{Ne}$ and $600^{\circ} \mathrm{C}$ for $\mathrm{D}$ plasma). Namely, higher temperature in the center means lower $D$ retention due to the larger probability for $D$ de-trapping and higher diffusion and increase of $D$ amount at the edge of Ne-D plasma beam due to increased surface modification and lower sample temperature.

In addition we analysed one crater on the surface that was produced by in laserinduced breakdown spectroscopy (LIBS) measurements. The LIBS measurements were performed inside of $1 \mathrm{~mm}^{2}$ regions with $\mathrm{Nd}$-YAG laser $(\lambda=1064 \mathrm{~nm})$ with surface fluence $16 \mathrm{~J} / \mathrm{cm}^{2}$ and pulse duration of $8 \mathrm{~ns}$ [19]. We divided the LIBS crater into 6 concentric regions (see Figure 8) and calculated the D areal density in the regions separately as shown in Figure 9. We observed that the amount of $D$ inside of LIBS crater is negligible as $D$ is almost totally desorbed during the LIBS measurements. A strong D depletion in the LIBS induced crater is also visible in Figure 7 at the position $X=-2000 \mu \mathrm{m}$.

Yields for heavier elements $\mathrm{W}$ and Mo obtained via 3HIXE on the Pilot PSI sample are shown in Figure 7. They are only approximately proportional to the concentration of the particular element due to absorption in the sample. Due to unknown exact ionizing cross sections for ${ }^{3} \mathrm{He}$ ions we were unable to quantify the results of $3 \mathrm{HIXE}$ measurements. However, qualitatively we observe a large increase of the Mo yield in the centre of crater. This can be explained with additional deposition of extra Mo from source and from the clamping rings that holds sample inside the Pilot-PSI machine. The opposite can be seen for $\mathrm{W}$. In the centre of crater we observe a decrease of $\mathrm{W}$ yield due to absorption of $\mathrm{W}$ x-rays in deposited Mo layer on top of the original $\mathrm{W}$ coating. Outside the crater region we observe uniform distribution of Mo and W. The RBS spectra (not shown) show a mixture of Mo and W layer on top 
of the surface what is not observed further away from the centre. Therefore the Mo signal increase is mainly due to Mo deposition on the layer that comes from the plasma source itself and possibly also due to small erosion of $\mathrm{W}$ layer in the centre. Results from our measurements agree well with the qualitative LIBS and SIMS observations done on the same PSI sample [19].

\section{Conclusion}

In this study we have analysed samples exposed in the ASDEX Upgrade tokamak and in the Pilot-PSI linear plasma device with a focused ${ }^{3} \mathrm{He}$ beam on the $\mu \mathrm{m}$ lateral resolution. The ${ }^{3} \mathrm{He}$ ion beam is used to determine the concentration of deuterium via the nuclear reaction analysis. Analyses were done using a $2 \mathrm{MV}$ tandem accelerator coupled with a duoplasmatron ion source. In the experimental chamber we were able to produce a focused ${ }^{3} \mathrm{He}$ beam with $10 \times 10 \mu \mathrm{m}^{2}$ beam size and an ion current of 300 pA at the energy of 3.3 MeV.

For AUG samples, we studied the effects of surface roughness on erosion and deposition of $\mathrm{W}$. The results show that in the case of unpolished graphite samples coated with tungsten, the erosion/deposition is increased near the strike point, but it is not very distinct. In the case of polished graphite samples there is a clear depletion of the $\mathrm{W}$ layer near the strike point. By detailed 3HIXE mapping we observe a "leopard" skin pattern on the polished surface, as large concentration of $\mathrm{W}$ is observed on carbon aggregates which are left on the surface after polishing. One could conclude from this that such aggregates are more susceptible to erosion. While the unpolished surface is less prone to erosion as its features protect the material via shadowed valleys.

For samples exposed in Pilot-PSI machine, we measured the deuterium amount around the central strike crater. Qualitatively we observe a large increase of the Mo yield in the centre of crater. This can be explained with additional deposition of extra 
Mo from source and from the clamping rings that hold sample inside the Pilot-PSI machine. The $\mathrm{W}$ yield is decreased in the center of the crater due to additional absorption of x-rays in the deposited Mo layers. Outside the crater region we observe uniform distribution of Mo and W. We found a good agreement between our measurements and SIMS and LIBS measurement. The D amount was also analysed in the centre of the LIBS craters, inside which we detected very low amounts of $D$. This confirms that the LIBS method is able to desorb the majority of deuterium in the analysed area and no deuterium was re-adsorbed during the ablation process.

While LIBS is a useful technique to measure the $D$ inventory of the inner walls of tokamaks there are still issues in quantifying the results. Therefore NRA provides a complementary information on the $D$ inventory. By comparing the LIBS and NRA results one can make educated guesses on the $D$ concentrations in inner wall during the tokamak operation. With these results we provide a new powerful analytical tool for elemental inventory of plasma facing materials.

\section{Acknowledgments}

This work has been carried out within the framework of the EUROfusion Consortium and has received funding from the Euratom research and training programme 20142018 under grant agreement No 633053. Work was performed under EUROfusion WP PFC. The views and opinions expressed herein do not necessarily reflect those of the European Commission.

\section{References}

[1] G. Federici et al, Nucl. Fusion 41 (2001) 1967

[2] S. Markelj et al, J. Nucl. Mater. 469 (2016) 133

[3] M. Mayer et al. Nucl. Instr. Meth. B 267 (2009) 506 
[4] S. Koivuranta et al, J. Nucl. Mater. 438 (2013) 735

[5] M. Rubel et al, , Nucl. Instr. Meth. B 371 (2016) 4

[6] M. Yamagiwa et al, Phys. Scripta 2011 T 145

[7] S. Markelj et al, Phys. Scripta 2014 T159

[8] A. Založnik et al, Phys. Scripta 2016 T167

[9] S. Markel et al, Deuterium Retention Studies in Self-ion Damaged Tungsten Exposed to Neutral Atoms, 24st International Conference Nuclear Energy for New Europe, Portorož, 2015

[10] P. Pelicon et al, Nucl. Instr. Meth. B 269 (2011), 2317

[11] H. Khodja et al, Nucl. Instrum.Meth. B 266 (2008) 1425

[12] P. Petersson et al., Nucl. Instr. Meth. B 268 (2010) 1833

[13] G. Federici et al, Nucl. Fusion 313 (2003) 11

[14] H. Zohm et al, Nucl. Fusion 55 (2015) 10

[15] A. Herrmann et al, Fusion Engineering and Design, 98-99 (2015’) 1496

[16] A. Hakola et al, Phys. Scripta 2016 T167

[17] G. J. van Rooij et al. J. Nucl. Mater. 415 (2011) 137

[18] P. Paris et al, J. Nucl. Mater 438 (2013) 754

[19] K. Piip et al, LIBS detection of erosion/deposition and deuterium retention resulting from exposure to Pilot-PSI plasmas, in preparation.

[20] P. Pelicon et al., Nucl. Instr. Meth. B 332 (2014) 229

[21] K. Vogel-Mikus et al. Nucl. Instr. Meth. B 267 (2009) 2884

[22] M. Mayer 1997 SIMNRA User's Guide, Report IPP 9/113,Max-Planck-Instutut für Plasmaphysik Garching

\section{List of figure captions}

Fig.1: Experimental set-up at micro-PIXE end station with the added NRA detector.

Fig. 2: Picture of the two samples exposed in ASDEX Upgrade (AUG). a) The unpolished graphite tile with $25 \mathrm{~nm}$ of deposited W. b) The smooth/polished graphite 
tile with $25 \mathrm{~nm}$ of initially deposited W. On both samples we marketed regions where micro-NRA and 3HIXE measurements were performed. Orientation of samples inside of AUG is same for both samples and is marked on figure $2 a$.

Fig. 3: Picture of sample exposed to deuterium plasma in Pilot PSI machine. The sample consists of $1.5 \mu \mathrm{m}$ coating of $95 \% \mathrm{~W}$ and $5 \% \mathrm{Y}$ on bulk Mo. On sample we marketed regions where micro-NRA and 3 HIXE measurements were performed. Central plasma strike crater is visible. Also three large craters are visible, which are produced by the LIBS measurements. Below our scanning areas series small craters are visible due to the SIMS measurements.

Fig. 4: Total amounts of $D$ and $W$ for both samples exposed in ASDEX Upgrade tokamak plotted in poloidal direction. The D amounts were calculated from NRA yields with SIMNRA [22] software. The W amounts were determined by RBS. Thickness was calculated by using density of $W \rho_{w}=19.29 \mathrm{~g} / \mathrm{cm}^{3}$ as provided by SIMNRA [22].

Fig. 5: PIXE measurements of W L-lines on polished (left - 819002) and unpolished (right - 819006) AUG sample. On smooth sample we see the deposition of W on C aggregates - W deposition like a "lepard-skin" patern.

Fig. 6: Images with secondary electrons done with scanning electron microscope (SEM) of AUG samples. On the left is the image of polished sample with visible $\mathrm{W}$ deposited on carbon aggregates, some marked with red circles as an example. On 
the right is image of unpolished graphite. The SEM images were done at Center for electron microscopy and microanalysis (CEMM) of Jožef Stefan Institute.

Fig. 7: Total amount of $D$ and 3 HIXE yields of $W$ and Mo are shown. Numbers on the top (1-9) correspond to specific regions shown on Figure 3 while origin of $x$-axis is in the center of Pilot-PSI plasma beam. We calculated D concentrations with SIMNRA [22] software.

Fig. 8: 2D distribution of $D$ yield around LIBS crater on Pilot PSI sample with marked regions inside the crater. Scales on axis are in $\mu \mathrm{m}$.

Fig. 9: Amount of D inside the LIBS crater on the Pilot PSI sample. Number on data point corresponds to the concentric region shown on Figure 8. 


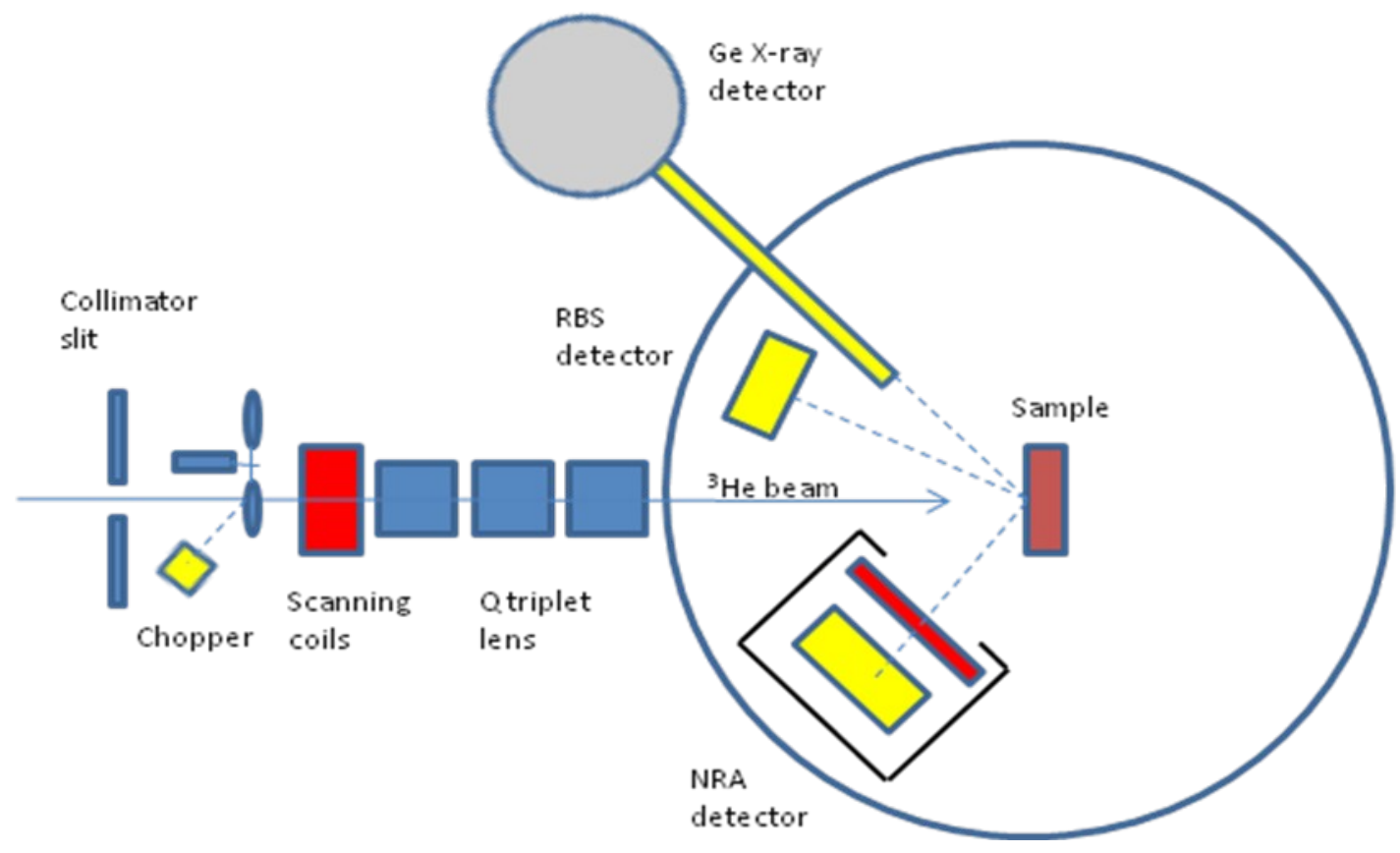

Figure 1

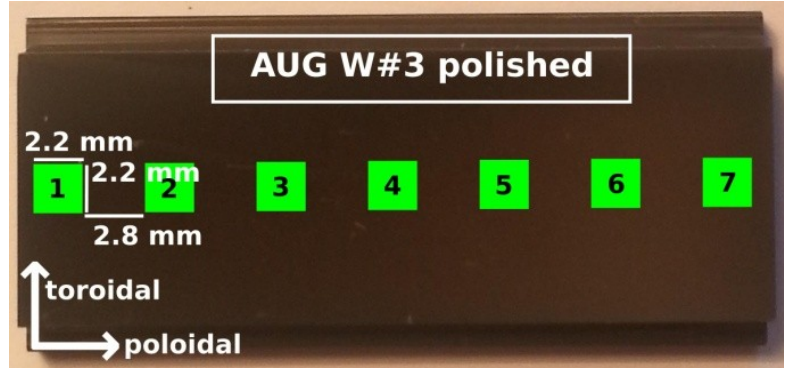

Figure $2 a$

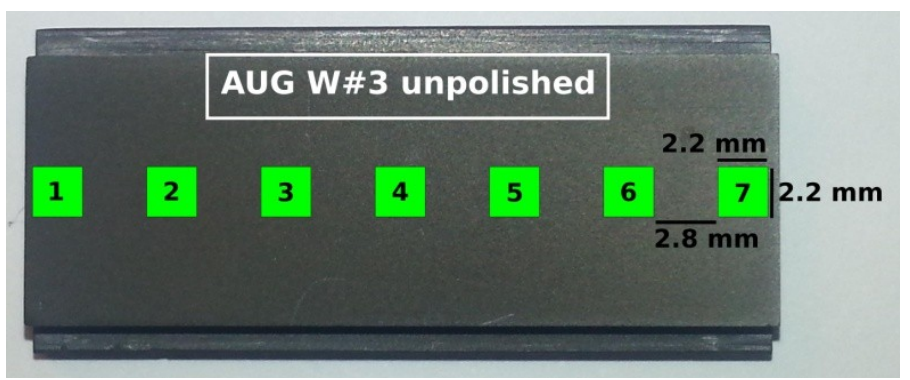

Figure $2 b$ 


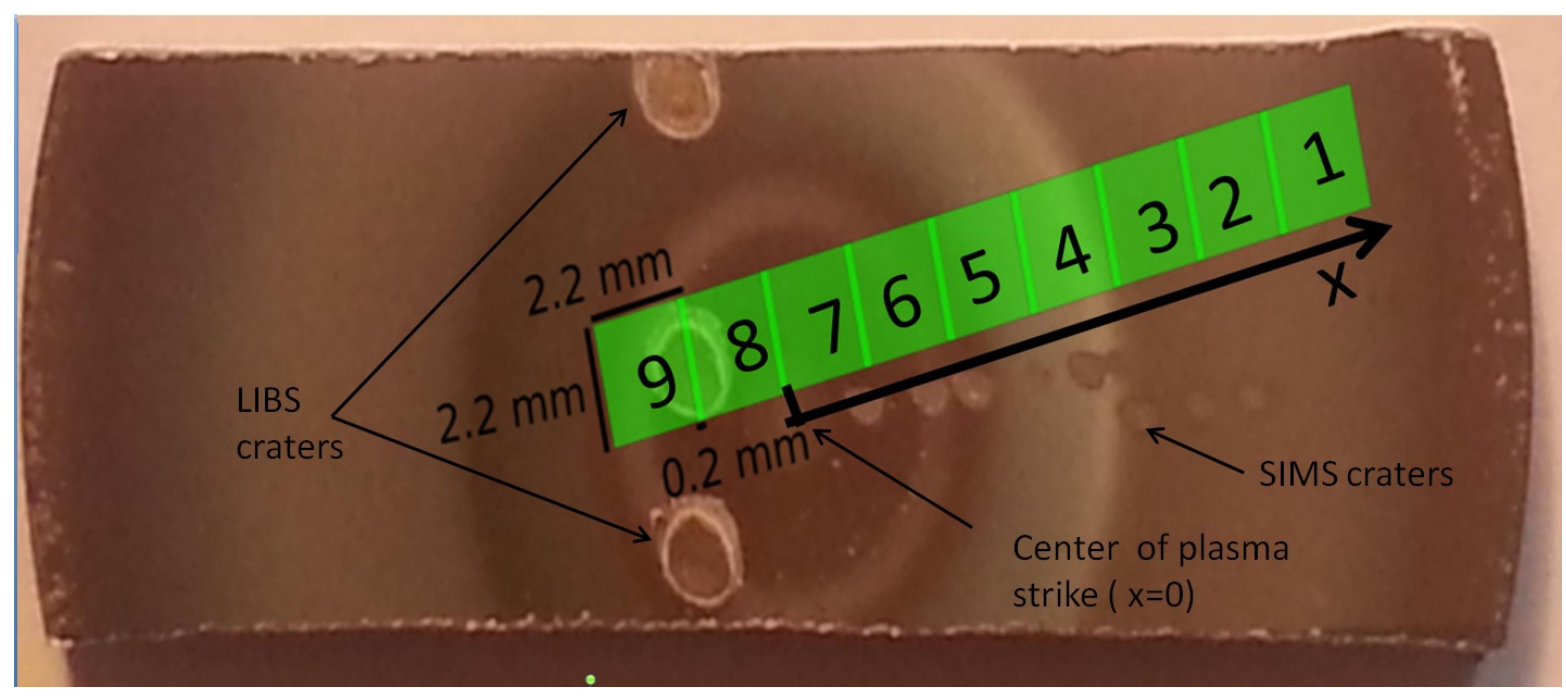

Figure 3

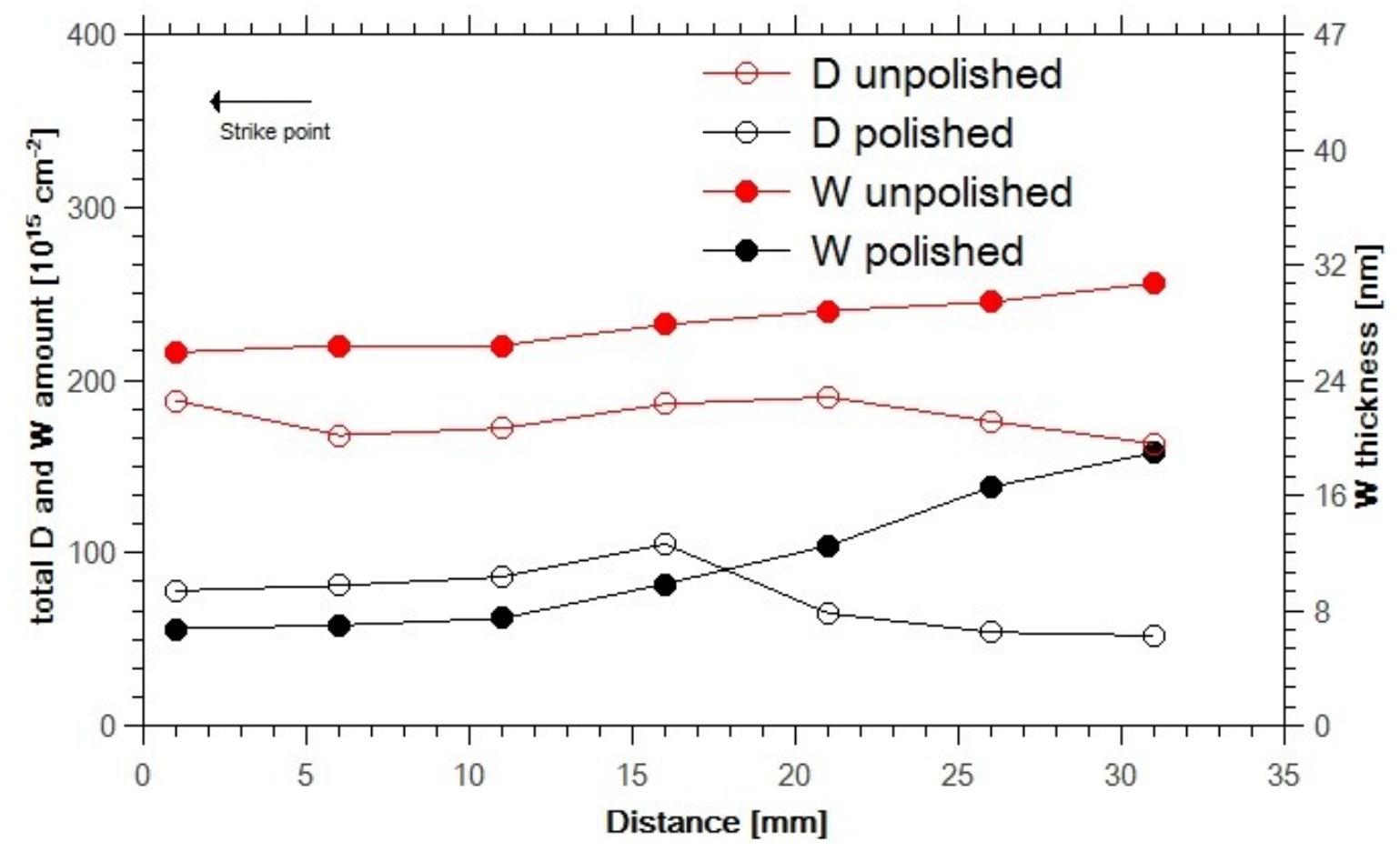

Figure4 

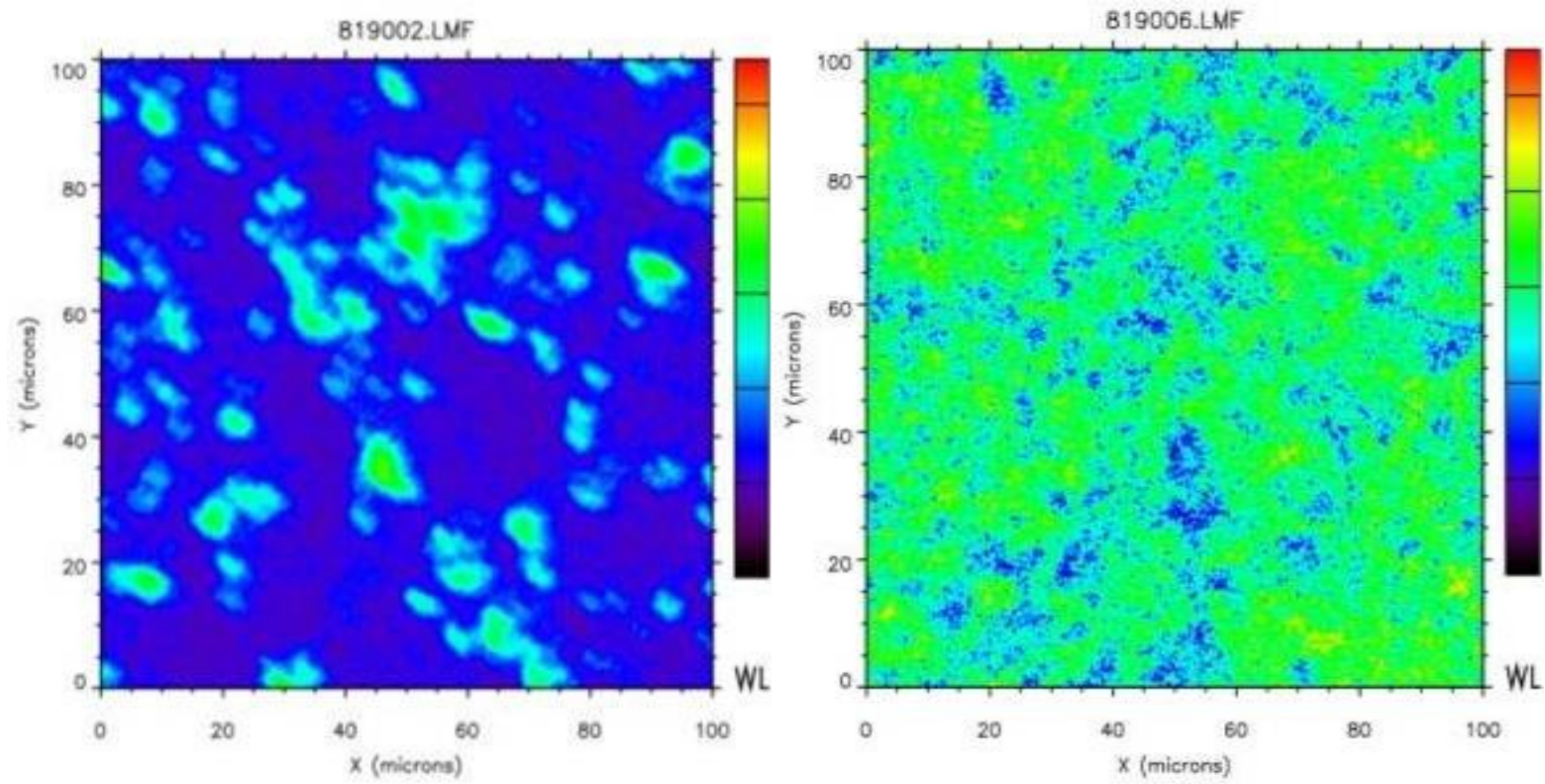

Figure 5

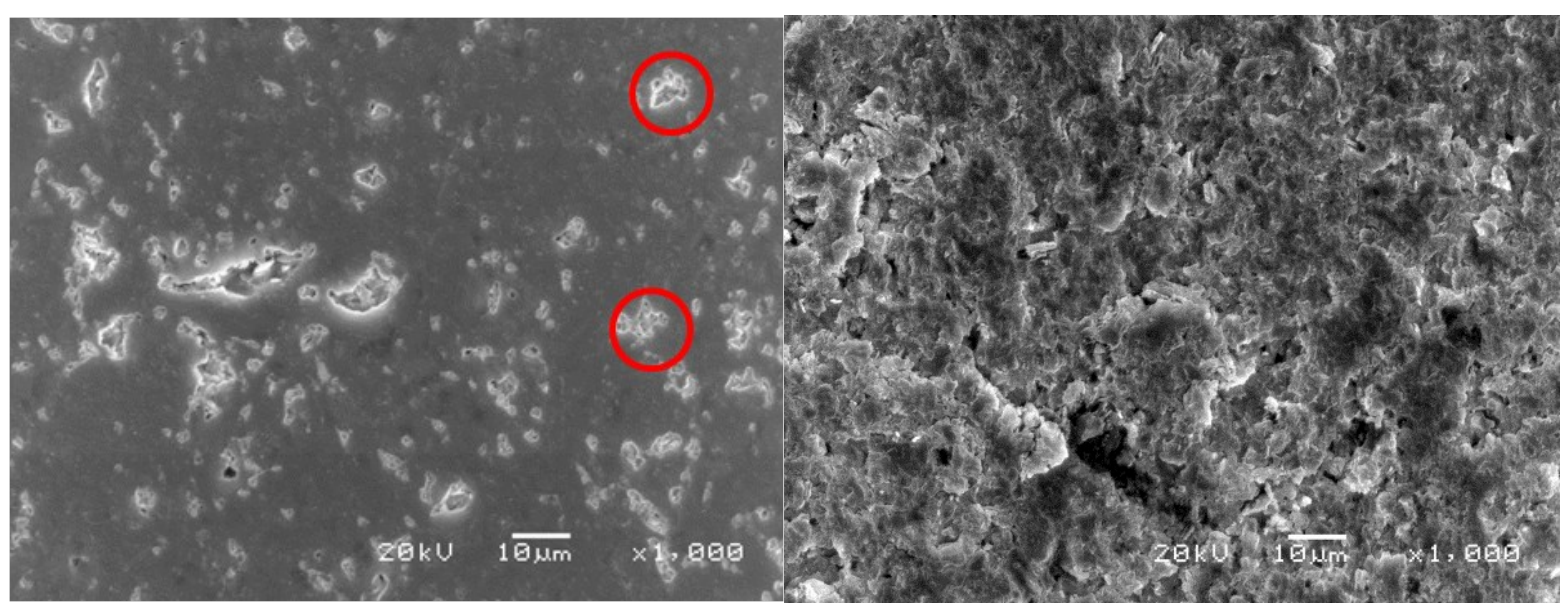

Figure 6 


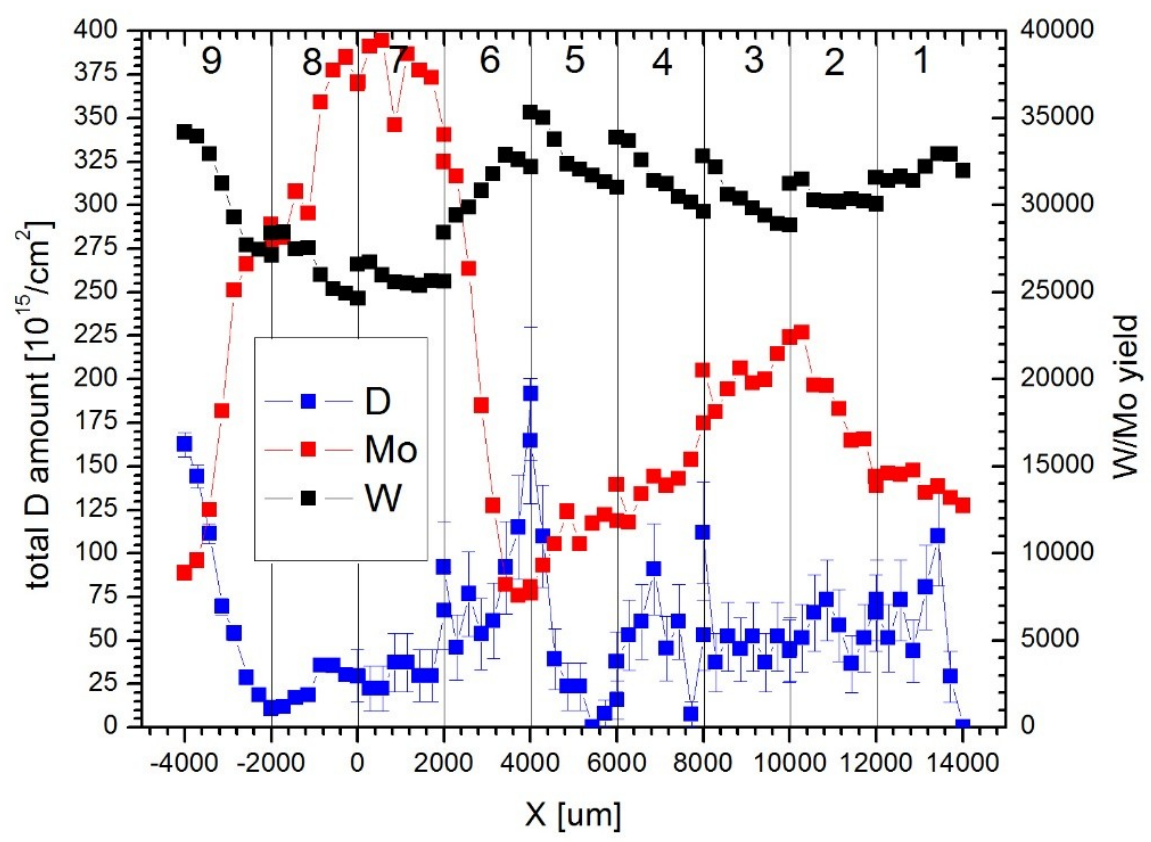

Figure7

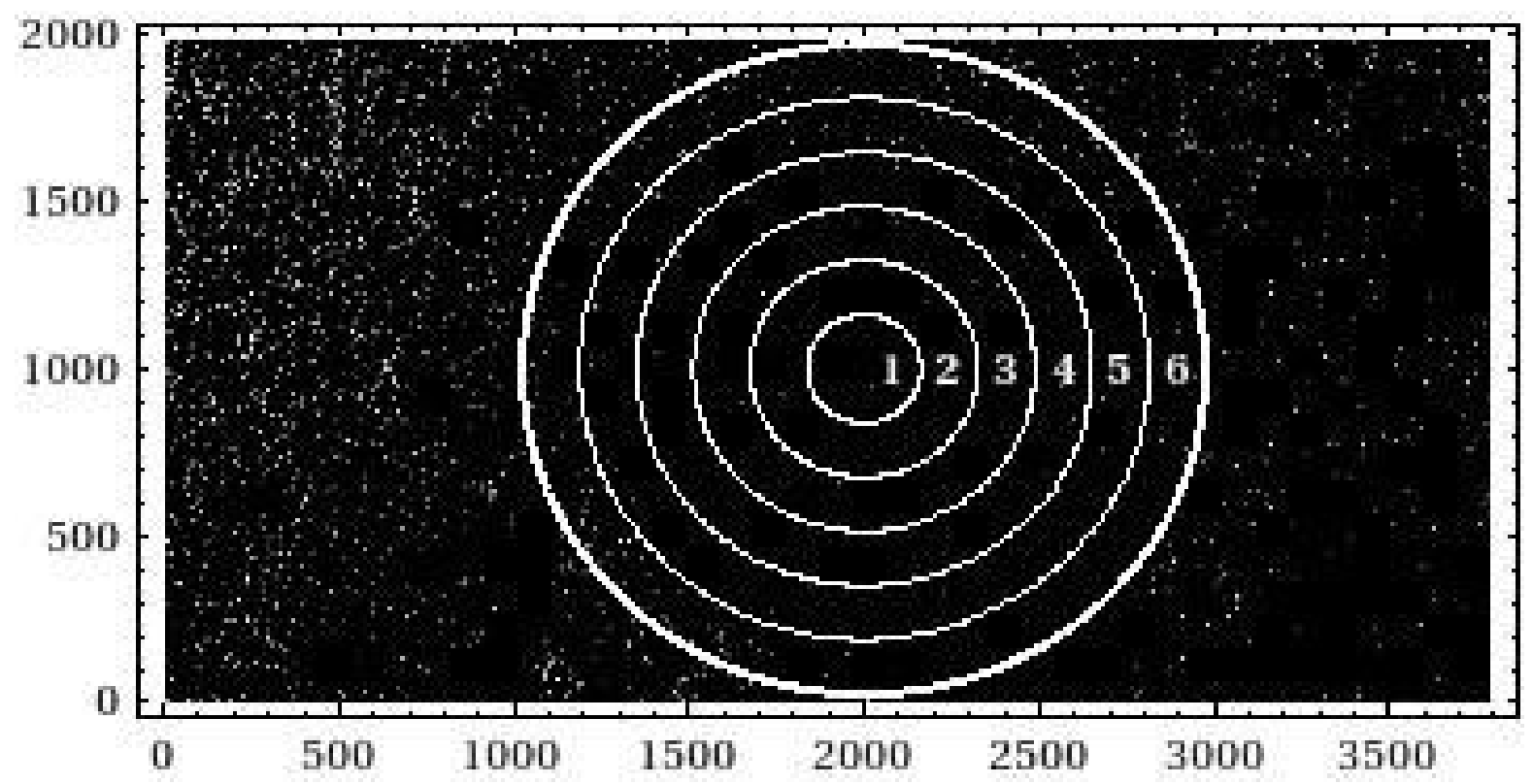

Figure 8 


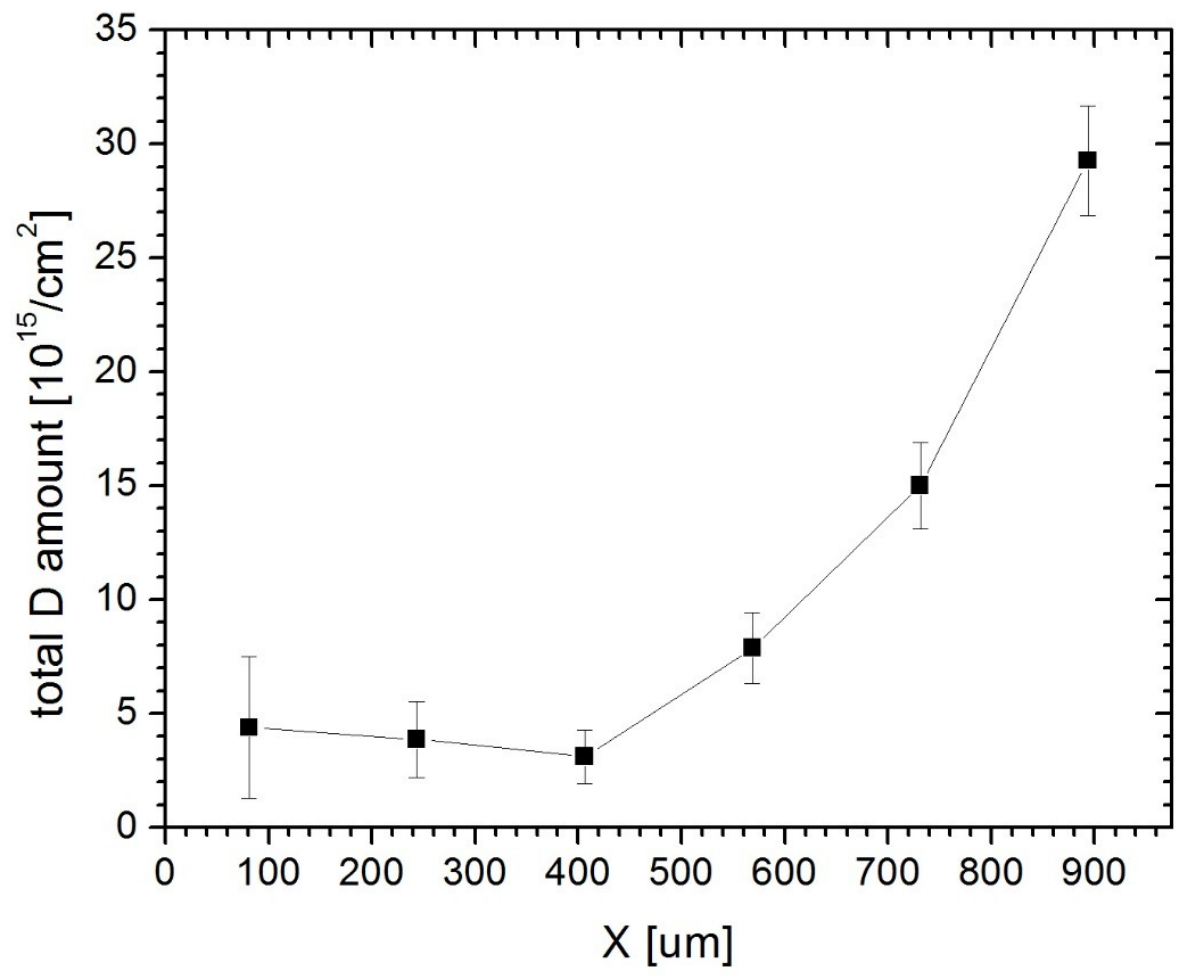

Figure 9 